\title{
Nursing Research on Comprehensive Intervention of Pleural Reaction Undergoing Thoracentesis
}

\author{
WANG Jun ${ }^{1}$, ZHAO Mengfen ${ }^{1}$,WU Jiani ${ }^{1}$, XU Zuofeng2* \\ Seventh Affiliated Hospital of Sun Yat-sen University \\ 1.Center of Excellence E-mail:727236396@qq.com.cn https://orcid.org/0000-0002-1170-8317 \\ 2. Ultrasound Department E-mail:xuzuofeng77@aliyun.com \\ https://orcid.org/0000-0003-3611-9698
}

Article History Received 13 June 2021 Accepted 25 July 2021 Published 30 September 2021 Cite this Article WANG Jun, ZHAO Mengfen,WU Jiani, XU Zuofeng. Nursing Research on Comprehensive Intervention of Pleural Reaction Undergoing Thoracentesis [J].Medical Research, 2021.3(3):81-87, http://dx.doi.org/mrhk/10.6913/MRHK.202109_3(3).0009

Copyright @ 2021 Creative Publishing Co., Limited. All rights reserved.Email:mrhk@mrhk.cc

\begin{abstract}
Objective To explore the influence of different postures for pleural reaction in patients undergoing thoracic interventional operations.
\end{abstract}

Methods The 695 patients with pleural effusion or hydro-pneumothorax underwent thoracic interventional operations (thoracentesis and thoracic catheter drainage) by ultrasound guidance from May, 2018 to February, 2021 were involved in this study. Two groups were divided in randomly, one was sitting posture group, 312 patients was preformed operations in this postures, the other group included 383 patients who were in supine or lateral positions. The pleural reaction rates of the two groups were compared.

Results The pleural reaction rates were $18.5 \%$ (58/312) in sitting posture group and $2.8 \%$ $(11 / 383)$ in supine group, there were statistically significant difference between the two groups.

Conclusion Postures of patients can affect the occurrence of pleural reaction, supine position can reduce the incidence of pleural reaction.

Key words Pleural effusion; Thoracentesis; Pleural reaction; Posture

The most serious complication of pleural puncture is pleural reaction, with the incidence rate of about $4.8 \%-20 \%$. It is also easy to be ignored in clinical nursing ${ }^{1,2,3}$. Among the 
causes of pleural reaction induced by pleural puncture, the first puncture, mental stress, fasting and elderly weakness are the most common. Pleural response was manifested as pale face, dyspnea, dizziness, chest tightness, nausea and vomiting, sweating, palpitation, and even shock $^{2,4}$. This paper summarizes the cases of ultrasound-guided pleural puncture (pleural puncture drainage and pleural puncture catheterization) compares the incidence of pleural reactions in patients with different postures (sitting position and supine position), analyzes the causes and summarizes the skills for preventing pleural reactions. The data was collected in May 2018 to February 2021 from the department of ultrasound of a hospital in Shenzhen, China.

\section{Methods}

\subsection{Data Collection}

695 patients who underwent ultrasound-guided pleural effusion drainage or pleural effusion catheterization in the Seventh Affiliated Hospital of Sun Yat-sen University from May 2018 to February 2021 were collected. Among them, 312 cases of pleural puncture were performed in sitting position, 198 males and 114 females, aged from 17 to 82 years, with an average age of 49 years \pm 0.5 years. There were 18 cases of pleural effusion drainage and 294 cases of simple pleural effusion catheterization. 383 cases of first thoracic puncture were performed in supine position, including 223 males and 160 females, aged 6 days +11 hours to 91 years old, with an average of $(48.0 \pm 1.4)$ years old. There were 11 cases of pleural effusion drainage and 372 cases of thoracic puncture catheterization and drainage. There was no significant difference in age and gender between the two groups.

\subsection{Treatment Methods}

All patients were punctured under ultrasound guidance, using $2 \%$ lidocaine $5 \mathrm{~mL}$ local anesthesia, using PTC (18G) puncture needle percutaneous puncture point into the chest, $10 \mathrm{~mL}$ syringe back to observe the presence of pleural effusion, and observe the color and character of the extract, then according to the purpose of puncture (diagnostic or therapeutic) choose single puncture drainage or catheter drainage. The speed of liquid extraction or drainage should not be too fast. The volume of liquid extraction or drainage should be less than $1000 \mathrm{~mL}$ per day.

\subsection{Prevention and Care}

Preparation for thoracic puncture should take the initiative to explain the purpose and significance of thoracic puncture to patients, explain the matters that patients need to pay attention to in the process of puncture do not rotate the body at will, relax the whole body, 
cooperate with the doctor to do rhythmic breathing, etc. in the preoperative patient should be patiently and carefully done to explain the risk of puncture, alleviate patient tension and supervise the doctor to sign informed consent. Patients with intraoperative tension can be appeased by talking to distract the attention of patients and playing soothing music. During the whole operation, the nursing staff should carefully observe the changes of vital signs of patients and effectively cooperate with doctors to complete the operation. Once the patient has pleural reaction symptoms, terminating the operation immediately and adjusting lying position, oxygen inhalation, ECG monitoring, oxygen saturation monitoring should be taken.

\subsection{Statistical Methods}

SPSS 21.0 software was used for statistical processing of all data. X2 test was used to compare the rate $(\%)$ between groups of enumeration data. If $\mathrm{P}<0.05$, the difference was statistically significant.

\section{Results}

2.169 cases of pleural reaction occurred in all 695 patients, with the incidence of $9.9 \%$. The incidence of pleural reaction in sitting group was higher than that in supine group, and the difference was statistically significant $(\mathrm{X} 2=47.5, \mathrm{P}<0.001)$. Comparison of the incidence of pleural reactions between the two groups is shown in table $\mathbf{1 .}$

Table 1 Compared the incidences of pleural reaction by thoracentesis with different positions

\begin{tabular}{ccccc}
\hline Group & Case number & Positive cases & Negative cases & Incidence of pleural reaction (\%) \\
\hline Sitting Group & 312 & 58 & 254 & 18.5 \\
Supine Group & 383 & 11 & 372 & 2.8 \\
\hline
\end{tabular}

2.2 The clinical manifestations of 69 patients with pleural reaction mainly included syncope, low blood pressure, cold sweating, dizziness, pale face, palpitation, and cold limbs. The incidence of different clinical manifestations of pleural reaction in the two groups was compared in table 2. The incidence of various clinical manifestations in the sitting group was higher than that in the supine group, and the difference between the two groups was statistically significant.

Table 2 Clinical manifestation of pleural reaction and the corresponding position

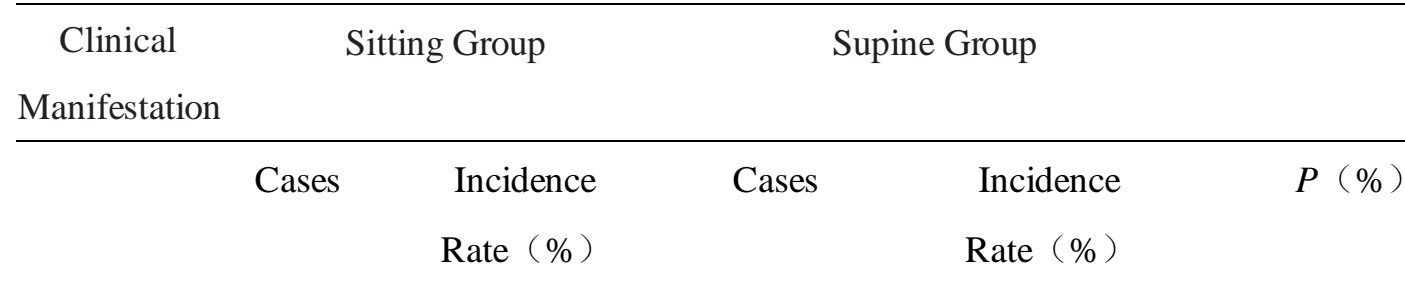




\begin{tabular}{cccccc}
\hline syncope & 18 & 5.8 & 0 & 0.0 & $<0.001$ \\
low blood & 12 & 3.9 & 0 & 0.0 & $<0.001$ \\
pressure & & & & & \\
cold & 31 & 9.9 & 3 & 0.3 & $<0.001$ \\
sweating & & 14.7 & 2 & 0.8 & $<0.001$ \\
dizziness & 46 & 15.4 & 0 & 0.5 & $<0.001$ \\
palpitation & 48 & 2.2 & 3 & $0.0 \%$ & $<0.001$ \\
pale face & 7 & 6.1 & $0.8 \%$ & \\
cold limbs & 19 & & & & $<0.001$ \\
\hline
\end{tabular}

\section{Discussion}

The complications of pleural puncture include pulmonary edema, pneumothorax, bleeding, and pleural reaction. Pleural reaction is a common complication of pleural puncture. The incidence rate reported in China is $9.7 \%-16.8 \% 5$. The complications of pleural puncture can affects the smooth of operation, and even cause shock, consequently endanger the life of patients. The causes may be related to the patient's basic diseases, psychology, and the professional and technical level of the intervention operator ${ }^{6}$.

In this study, once the occurrence of pleural stress reaction, the nursing staff kept the patients warm in time. After the posture was changed to supine position, most symptoms were relieved, and the clinical manifestations of pleural reaction in supine position were mild, without syncope and hypotension. If the patient is unable to lie supine or laterally due to disease, the bed head was raised by $30^{\circ}-45^{\circ}$ to relieve symptoms. Seizure and hypotension were found in sitting position. After changing the position, the symptoms of 42 cases were relieved in 1-3 min, including 5 cases of dizziness and sweating. After changing the position to supine position, the symptoms were relieved in 4-5 min. Pleural puncture was successfully completed in lateral position, and the clinical symptoms of supine position were mild. When stress reaction occurs in thoracic puncture in supine position, the reduction of cardiac blood volume caused by vasoconstriction is not significantly related to sitting position. The incidence of pleural reaction in supine group was lower than that in sitting group. Ye et al. $^{7}$ reported that when 175 cases of pleural effusion were examined by thoracoscopy in lateral position, only 3 cases had pleural reaction, with the incidence rate of $0.4 \%$. Therefore, it was speculated that reasonable application of analgesic and sedative drugs to reduce the pain sensitivity of patients and lateral position could reduce the occurrence of pleural reaction. 
This study point that the most common manifestations of pleural reaction are palpitation, dizziness and cold sweating. Timely adjustment of position to supine position can effectively prevent the occurrence of adverse reactions. Among 58 patients with pleural reaction in sitting position, 12 cases of dizziness and cold sweating were relieved after posture change and continued pleural puncture operation in lateral position, which suggest that lateral position should be the first choice to adjust the position.

During the operation, patients were asked to breathe moderately and stably, which could reduce the pleural reaction. For patients with weak body, semi-lateral position could reduce the occurrence of pleural reaction. As far as possible, patients should not be subjected to fasting puncture. When emergency chest puncture needed in fasting patients, pleural reactions can occur, such as progressive decline in blood pressure, pale face, chest pressure, etc. The operation should be stopped immediately, so that the patients are in a supine position. They should be given sufficient warmth, inhaled oxygen, and continuous dynamic monitoring of the vital signs of the patients. Patients with mild symptoms can relieve themselves after oxygen inhalation and psychological nursing. Some patients with severe symptoms can be recovered after intravenous injection or oral administration of $20 \mathrm{ml} 50 \%$ glucose or a small amount of timely feeding. After recovery, they should follow the doctor's advice for symptomatic treatment. The patients in our department are mainly hospitalized patients, and the amount of thoracic puncture in outpatient is small, which is not included in this study. Before operation, the monitoring of coagulation blood routine, blood routine and nucleic acid was improved, and the routine feeding was performed. Patiently listen to the patient's complaints to achieve early detection and timely treatment.

Nursing intervention of posture change in the process of thoracocentesis bring convenience and comfort through and affect the success rate of puncture ${ }^{5,7}$. With the vigorous development of increasing thoracic puncture into drainage tube, if the operation is terminated immediately caused by pleural reaction occurs, It's not only delay the diagnosis and treatment of patients, but also result in a waste of medical resources. Thus, reducing the incidence of pleural reaction is of social value ${ }^{8}$. To reduce the incidence of pleural reaction, many scholars have conducted valuable research, such as music therapy, narrative nursing, and ondansetron ${ }^{8,9,10}$. Through this study, we found that supine position could reduce the occurrence of pleural reaction. At present, most thoracic punctures are still routinely performed in upright 
position $^{11,12}$. Whether the selection of supine position can significantly reduce the occurrence of pleural reaction and promote it in clinic to solve clinical and nursing practical problems remains to be further confirmed by prospective controlled nursing research. Based on the results of our study, in patients who must be seated, medical staff should improve preoperative preparation in advance, such as : strengthening sedation, analgesia, emergency preparedness, in case of difficult puncture in the anesthesia department to help complete ${ }^{13}$. Therefore, for patients with thoracic puncture, comprehensive nursing intervention, including:posture change, psychological nursing can not only reduce the occurrence of postoperative complications, but also improve the quality of life of patients.

\section{Reference:}

1. Cantey Eric P,,Walter James M,,Corbridge Thomas \& Barsuk Jeffrey H.(2016).Complications of thoracentesis: incidence, risk factors, and strategies for prevention.. Current opinion in pulmonary medicine(4), doi:10.1097/MCP.0000000000000285.

2. LARGHERO, P. (1961). Anterior High Thoracocentesis in a Downward Prone Position. El Torax, 10, 289-290.

3. Sagar Ala Eddin S., Landaeta Maria F., Adrianza Andres M., Aldana Grecia L., Pozo Leonardo, Armas Villalba Aristides... \& Jimenez Carlos A.. (2020).Complications following symptom-limited thoracentesis using suction. European Respiratory Journal (5), doi:10.1183/13993003.02356-2019.

4. Elyah Oren \& Chatterji Sumit. (2020). A Review of Symptoms and Complications of Ultrasound Assisted Thoracentesis in the First Specialist Pleural Clinic in Israel.. The Israel Medical Association journal : IMAJ(12), doi: 2008(3):32-34.

5. Cao, W., Wang, Y., Zhou, N., \& Xu, B. (2016). Efficacy of ultrasound-guided thoracentesis catheter drainage for pleural effusion. Oncology letters, 12(6), 4445-4448.

6. Ferreiro, L., Lado-Baleato, Ó., Toubes, M. E., Suárez-Antelo, J., Pose-Reino, A., San José, M. E., Álvarez-Dobaño, J. M., González-Barcala, F. J., Ricoy, J., Gude, F., \& Valdés, L. (2020). Identification of Pleural Response Patterns: A Cluster Analysis. Archivos de bronconeumologia, 56(7), 426-434. https://doi.org/10.1016/j.arbres.2019.08.021.

7. Ye Y., Liu J., \& Lin L., (2012). Common adverse reactions and treatment of medical thoracoscopy ( analysis of 175 cases ). Chinese Journal of Endoscope. 12(1) : 101-103.

8. Twose Chloe,Ferris Rebecca,Wilson Andrew,Rahman Najib,Farquhar Morag \& Mishra Eleanor.(2021).Therapeutic thoracentesis symptoms and activity: a qualitative study. BMJ Supportive \& Palliative Care(), doi:10.1136/BMJSPCARE-2020-002584.

9. Wang Y., Liao J., \& Jiang P. (2016). Ondansetron for the prevention of pleural reaction after thoracic puncture. Modern medical and health, 32 (20) : 3206-320.

10. Wang J. (2016). Observation on the effect of music therapy on reducing pleural 
reaction in thoracic puncture. General nursing, (23) : 2408-2409.

11. Brandstetter, R. D., Velazquez, V., Viejo, C., \& Karetzky, M. (1994). Postural changes in pleural fluid constituents. Chest, 105(5), 1458-146.

12. Jin, K. N., Ko, J. M., Kim, J., Ahn, M. I., Kim, S. C., \& Han, D. H. (2014). Novel supine thoracentesis bed enabling thoracentesis via the posterior axillary line. Cardiovascular and interventional radiology, 37(3), 805-809. https://doi.org/10.1007/s00270-013-0763-3.

13. Avasarala Sameer K \& Lentz Robert J.(2021).Reason for Consult: Still on Oxygen, Discharge Pending, Requesting Thoracentesis.. Journal of bronchology \& interventional pulmonology(3), doi:10.1097/LBR.0000000000000778. 